\title{
Bistable beam propagation in liquid crystals
}

\author{
Armando Piccardi*, Alessandro Alberucci, Nina Kravets, Oleksandr Buchnev, Gaetano Assanto, Fellow, IEEE
}

\begin{abstract}
Light-controlling-light is one of the most advanced paradigms in optical signal processing, including light-induced waveguides as well as all-optical switching and routing. Other fundamental aspects of all-optical processing are optical memories and sequential elements, which require responses depending on the evolution history of the system, such as the hysteresis stemming from optical multistability. Hereby we report on optical bistability and hysteresis in cavity-less geometries and in the presence of self-localized beams, i.e., spatial optical solitons, exploiting the nonlocal reorientational nonlinearity of nematic liquid crystals. When the optic axis of NLC is initially orthogonal to the applied electric field, the molecular dipoles start to rotate above a threshold named after the Fréedericksz transition, usually second-order. Here we show that such transition becomes first-order via the intrinsic feedback provided by self-focusing, in turn leading to the appearance of a hysteresis loop between diffracting and self-confined beams. We report on hysteresis of the beam size versus the input power, as well as hysteresis versus applied voltage at a fixed beam power. Our findings introduce a novel kind of cavity-less optical bistability with propagating light beams and disclose a novel approach to information storage based on light self-localization.
\end{abstract}

Index Terms-Bistability, Spatial solitons, Reorientational nonlinearity.

\section{INTRODUCTION}

Optical multistability occurs when light-matter interactions support the coexistence of two or more stable states sharing the same set of excitation parameters [1]. When exploiting dispersive and absorptive mechanisms [2], [3], optical multistability is usually due to the interplay of a nonlinear process and a feedback mechanism: the former stems from the material response to light, the latter can be inherent to the system or provided externally (e.g., by a cavity or a mirror). In the simplest limit of two stable states, optical bistability (OB) has been widely investigated because of its potentials for the implementation of optical memory elements and/or sequential logic circuits [4], [5], particularly in the framework of alloptical signal processing [6], [7], [8], [9], [10], [11], [12]. The vast available literature on $\mathrm{OB}$ includes, besides resonators and external mirrors [1], interfaces between nonlinear media [13], photonic crystals and distributed feedback gratings [14], [15], [16], [17], [18], [19], [20], [21], [22], distributed

A. Piccardi (email: armando.piccardi@uniroma3.it) is with NooEL - Nonlinear Optics and OptoElectronics Laboratory, University Roma Tre, 00146, Rome, Italy. * Corresponding author

A. Alberucci is with Optics Laboratory, Tampere University of Technology, FI-33101, Tampere, Finland

N. Kravets was with NooEL - Nonlinear Optics and OptoElectronics Lab, University Roma Tre, 00146, Rome, Italy

O. Buchnev is with Optoelectronics Research Centre, University of Southampton, SO17 1BJ, Southampton, United Kingdom

G. Assanto is with NooEL - Nonlinear Optics and OptoElectronics Lab, University Roma Tre, 00146, Rome, Italy and Optics Laboratory, Tampere University of Technology, FI-33101, Tampere, Finland coupling to waveguides [23], [24], [25], [26], nonlinear optical nanocavities and ring resonators [27], [28], graphene [29], Anderson localization [30], self-focusing with counterpropagating beams [31]. The self-focusing case corresponds to a distributed self-lens resulting from an intensity-dependent refractive index, as in the so-called Kerr or Kerr-like media, where such an intensity dependence is linear or nearly-linear. When self-focusing is strong enough to balance the natural beam spreading due to diffraction, the transverse profile of a light beam can remain invariant in propagation, corresponding to the fundamental mode of the self-induced waveguide [32]. Such self-trapped wavepackets are named spatial solitons. Spatial solitons (and their temporal counterparts where selfphase modulation compensates chromatic dispersion) have been widely investigated in optics during the last decades for their ubiquitous character [33], [34]. The possibility of combining light self-localization into solitons with $\mathrm{OB}$ was investigated theoretically by Kaplan in 1985 [35], but never demonstrated experimentally until 2014, when it was reported by us in liquid crystals without external feedback [36].

In this Paper we address, both experimentally and theoretically, OB between wave-propagation states corresponding to beam diffraction and spatial solitons, respectively, in an optically nonlinear as well as electro-optic reorientational medium, namely nematic liquid crystals (NLC), where either the optical power or the bias voltage can control light localization and its evolution/switching between different states. With respect to our previous works [36], [37], here we generalize the approach of Ref. [38] developing a general theory based upon the Green function, and accounting for the simultaneous presence of voltage and optical fields. Our semi-analytic approach provides a simple physical picture of the central role played by self-focusing in the appearance of optical bistability in the highly nonlocal limit. We also address the role played by the longitudinal beam variation, a point never dealt with in detail in the previous works.

Liquid crystals are soft materials sharing the properties of both solids and liquids; in the nematic phase, the elongated organic molecules are randomly distributed in space with a high degree of orientational order, with the average direction of their long-axes associated to a point-wise unitvector distribution, the so called molecular director $\mathbf{n}$ [39]. Owing to such angular order, NLC are optically birefringent due to the anisotropic polarizability of their molecules, with refractive index eigenvalues $n_{\perp}$ and $n_{\|}$corresponding to electric fields perpendicular and parallel to the director, respectively. When uniformly aligned, most NLC behave as positive uniaxial dielectrics, with optic axis along $\mathbf{n}$, ordinarywave index $n_{o}=n_{\perp}$ and extraordinary-wave index $n_{e}(\theta)=$ $\left(\frac{\cos ^{2} \theta}{n_{\perp}^{2}}+\frac{\sin ^{2} \theta}{n_{\|}^{2}}\right)^{-1 / 2}$, depending on the angle $\theta$ between 
the director and the wave-vector of the propagating wave or wavepacket. The well established technology of NLC display and photonic devices exploits their peculiar optical properties and response to electromagnetic fields [40], including extended spectral transparency, high nonlinearity and electrooptic effect, easy tunability to external excitations as well large damage threshold [41]. The most exploited response to electric fields at both low and optical frequencies is reorientational: the molecular dipoles induced by electric fields react with a torque, which tends to reduce the overall system energy by aligning the director to the field vector. The elastic response, due to intermolecular interactions in the liquid crystalline phase, counteracts such torque and determines the final equilibrium, i.e., the resulting director distribution. In the case of an extraordinary polarized optical field, self-focusing can occur at low powers through the refractive index increase associated to molecular reorientation [42], [43]. A finite size light-beam can therefore reorient the NLC molecules and induce a graded index transverse profile, thus acting as a self-waveguide through compensation of linear diffraction: a self-confined wavepacket or spatial optical soliton in NLC is usually referred to as a "Nematicon" [44], [45], [46]. The reorientational nonlinearity of NLC is accompanied by a highly nonlocal response, with range depending only on the sample geometry in the unbiased case; nonlocality is an important stabilization mechanism of $(2+1) \mathrm{D}$ solitons against catastrophic collapse [47], [48]. Since the nonlinear response depends on the mutual alignment of molecular director and electric field, the torque vanishes when director and field vector are mutually perpendicular, i.e. $\theta=0^{\circ}$. In this limit the molecules, in the presence of noise, can only rotate above a power threshold, termed Fréedericskz transition (FT) [49]. The latter transition is usually second-order, i.e. it does not support bistability; nevertheless, under specific conditions on NLC properties or external feedback, first-order transitions with bistability were reported in NLC [50], [38], [51], [52], [53], [54], [55], [56]. In this work we investigate cavity-less first-order transitions in a standard material and configuration, exploiting the intrinsic feedback provided by the medium response to intense beams in order to obtain a bistable behavior without external feedback.

The Paper is structured as follows. In Sec. II we introduce the theoretical model and discuss molecular orientation featuring or not self-focusing. We demonstrate the evolution from second- to first-order transitions when self-focusing is strong enough to affect the beam profile. We also show how optical and electric fields interact in allowing/tuning a bistable behavior. In Sec. III we experimentally demonstrate the appearance of hysteresis loops between diffracting and self-trapped beams as predicted in Sec. II. Two kinds of loops can be observed: for a fixed bias voltage and varying the input beam power, for a fixed input beam power and varying the voltage. We also briefly address the role of temperature. Finally, in Sec. IV we summarize our results.

\section{BISTABLE BEAM STATES IN NEMATIC LIQUID CRYSTALS}

\section{A. Geometry and model}

The geometry we consider for the analysis and observation of $\mathrm{OB}$ is sketched in Fig. 1. The NLC sample consists of a planar cell defined by glass substrates infinitely extended along $y$ (a few cms in actual samples), of length $L_{z}$ along the propagation coordinate $z$ and thickness $h\left(h \ll L_{z}\right)$ across $x$. The glass/NLC interfaces are treated so to ensure a homogeneous director distribution in the absence of external stimuli, with planar alignment at $\theta=0^{\circ}$ [41]. On each of the interfaces parallel to the plane $y z$, Indium Tin Oxide thin film electrodes are deposited, permitting the application of a (low-frequency) bias voltage $V$ across the thickness $x$. To first approximation, the bias electric field $\mathbf{E}_{\mathrm{LF}}$ across the sample can be considered uniform, with $\mathbf{E}_{\mathrm{LF}}=\hat{x} V / h$ [40]. The optical excitation is a beam with electric field $\mathbf{E}_{\text {opt }}$ polarized along $\hat{x}$ and wavelength $\lambda$ with input wave-vector k $\|$ z. Hence, both the electric fields $\mathbf{E}_{\mathrm{LF}}$ and $\mathbf{E}_{\mathrm{opt}}$ tend to induce molecular rotation in the plane $x z$, so that the angle $\theta$ is sufficient to describe the director distribution in the medium. The reorientational torque due to an electric field is $\boldsymbol{\Gamma}=\epsilon_{0} \Delta \epsilon\langle(\boldsymbol{n} \cdot \boldsymbol{E})(\boldsymbol{n} \times \boldsymbol{E})\rangle$, where $\Delta \epsilon=\epsilon_{\|}-\epsilon_{\perp}$ is the anisotropy computed at the frequency of the external electric field $\boldsymbol{E}$. For a fixed field and director distribution, the torque diminishes for increasing temperature, vanishing at the isotropic-nematic transition. [39] The sample encompasses Fréedericksz transition as both low-frequency and opticalfrequency electric fields are initially orthogonal to the director. The director distribution is modified by the electromagnetic waves and described by the point-wise reorientation $\theta(x, y, z)$ resulting from the balance between the overall induced torque and the restoring elastic forces [39]:

$$
\nabla^{2} \theta+\frac{\epsilon_{0}}{2 K}\left[\frac{\epsilon_{a}\left|E_{\mathrm{opt}}(x, y, z)\right|^{2}}{2}+\Delta \epsilon_{L F} E_{\mathrm{LF}}^{2}\right] \sin 2 \theta=0
$$

where $\epsilon_{0}$ is the vacuum dielectric constant, $K$ is a scalar (effective) Frank's constant for the elastic response; $\epsilon_{a}=n_{\|}^{2}-n_{\perp}^{2}$ and $\Delta \epsilon_{L F}$ are the optical and low-frequency anisotropies, respectively. In Eq. (1) and in the following we neglect birefringent walk-off, that is, we take $\mathbf{E}_{\text {opt }} \| \hat{x}$ even after reorientation [57].

While the quasi-static field $\mathbf{E}_{\mathrm{LF}}$ is nearly independent of $\theta$ perturbations, the optical field distribution strongly depends on reorientation, as the latter leads to self-focusing and eventually self-localization. Specifically, naming $\theta_{m}(z)$ the maximum $\theta$ in each section $z=$ constant, paraxial light propagation in our case can be modeled by a nonlinear Schrödinger equation [36]:

$$
2 i k_{0} n_{e}\left(\theta_{m}\right) \frac{\partial A}{\partial z}+D_{x} \frac{\partial^{2} A}{\partial x^{2}}+\frac{\partial^{2} A}{\partial y^{2}}+k_{0}^{2} \Delta n_{e}^{2}(\theta)|A|^{2}=0,
$$

where $k_{0}$ is the vacuum wavenumber, $D_{x}$ is the diffraction coefficient along $x, \Delta n_{e}^{2}(\theta)=n_{e}^{2}(\theta)-n_{e}^{2}\left(\theta_{m}\right)$ is the extraordinary-wave refractive index well associated to the director distribution, $A=E_{\mathrm{opt}} e^{-i k_{0} n_{e}\left(\theta_{m}\right) z}$ is the slowlyvarying envelope of the beam. 
(a)
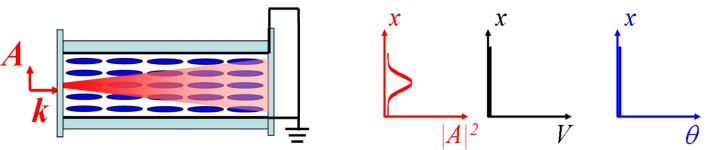

(b)
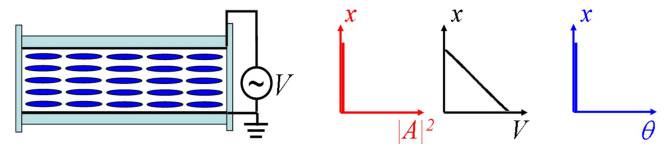

(c)
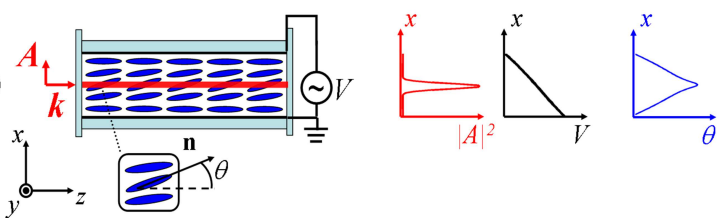

Fig. 1. Sketch of the NLC sample subject to FT. In a planar sample, reorientation is provided by either a light beam or an electric bias. To observe hysteresis in this work, FT is overcome by the combined action of both a light beam of envelope $A$ propagating along $z$ and an applied voltage $V$ across $x$. (a) A low-power beam (power below optical FT) diffracts due to lack of reorientation. (b) A modest external voltage (below electric FT) is not enough to reorient the molecules on its own. (c) The combined action of both low-frequency and optical frequency electric fields can induce molecular reorientation and result into beam self-confinement (nematicon). In each row, the corresponding profiles of beam intensity, voltage distribution and director orientation are sketched versus $x$, respectively.

\section{B. Molecular reorientation for constant excitations}

Equation (1) is a nonlinear equation in $\theta$ : thus, in general, the superposition principle cannot be applied to separate the effects of $\mathbf{E}_{\mathrm{LF}}$ and $\mathbf{E}_{\mathrm{opt}}$ [58]. Moreover, Eq. (1) must be solved with the boundary conditions $\theta=0^{\circ}$ on the glass/NLC interfaces, in line with the strong anchoring approximation. A vanishing $\theta(x, y, z)=0$ everywhere is always a solution of Eq. (1), regardless of the forcing terms; however, if the external excitations overcome the FT, non-trivial solutions exist. The Fréedericksz transition can be either first- or second-order, depending on the configuration, but bistability requires a first-order transition [6]. Here we are interested in optical bistability related with light self-focusing inside the NLC volume [50], [36].

1) Invariant beam profile: Let us start by considering solutions of Eq. (1) in a two-dimensional geometry, that is, neglecting the beam evolution along $z$ which is dictated by the interplay of diffraction and self-focusing. We assume that the beam intensity $I_{\mathrm{opt}}=\frac{n_{e}\left(\theta_{m}\right)}{2 Z_{0}}\left|\mathbf{E}_{\mathrm{opt}}\right|^{2}\left(Z_{0}\right.$ is the vacuum impedance) does not change with reorientation ${ }^{1}$. An invariant $I_{\text {opt }}$ entails a change in the optically-induced torque acting on the molecules, due to the non-homogeneous impedance of the medium: since for large anisotropies such mechanism can lead to bistability even in the absence of self-focusing [38], we need to account for it. Using the Green function formalism, $\theta$

\footnotetext{
${ }^{1}$ In the exact case, a Poisson equation should be solved jointly with Eq. (1), leading to a change in $\mathbf{E}_{\mathrm{LF}}$ with reorientation. In this Paper we neglect this second-order effect.
}

is formally given by

$$
\begin{gathered}
\theta(x, y)=\int_{-\infty}^{\infty} \int_{-h / 2}^{h / 2} G\left(x, y, x^{\prime}, y^{\prime}\right) \sin \left[2 \theta\left(x^{\prime}, y^{\prime}\right)\right] \times \\
{\left[\gamma_{\mathrm{opt}} I_{\mathrm{opt}}\left(x^{\prime}, y^{\prime}\right)+\gamma_{\mathrm{LF}} E_{\mathrm{LF}}^{2}\right] d x^{\prime} d y^{\prime},}
\end{gathered}
$$

where $\gamma_{\mathrm{LF}}=\epsilon_{0} \Delta \epsilon_{\mathrm{LF}} /(2 K), \quad \gamma_{\mathrm{opt}}\left(\theta_{m}\right)=$ $\epsilon_{0} \epsilon_{a} Z_{0} /\left[2 n_{e}\left(\theta_{m}\right) K\right]$, and the Green function $G$ in our geometry is [59]

$$
\begin{aligned}
& G\left(x, y, x^{\prime}, y^{\prime}\right)=\sum_{l=1}^{\infty} \frac{1}{\pi l} \sin \left[\frac{\pi l}{h}\left(x-\frac{h}{2}\right)\right] \times \\
& \sin \left[\frac{\pi l}{h}\left(x^{\prime}-\frac{h}{2}\right)\right] e^{-\frac{\pi l\left|y-y^{\prime}\right|}{h}} .
\end{aligned}
$$

Equation (3) is the starting point to look for approximate solutions of Eq. (1). Let us focus on the maximum reorientation $\theta_{m}$ [38]: if the optical intensity profile is circularly symmetric with respect to the cell axis $(x=0, y=0)$, to first approximation we can assume $\theta(x, y) \approx \theta_{m}=\theta(x=0, y=0)$ on the right hand side (RHS) of Eq. (3). We find

$$
\theta_{m}=F\left(I_{\mathrm{opt}}, \theta_{m}, V, h\right) \sin \left(2 \theta_{m}\right),
$$

where we defined $F\left(I_{\mathrm{opt}}, \theta_{m}, V, h\right)$ $\int_{-\infty}^{\infty} \int_{-h / 2}^{h / 2} G\left(0,0, x^{\prime}, y^{\prime}\right)\left(\gamma_{\mathrm{opt}} I_{\mathrm{opt}}+\gamma_{\mathrm{LF}} E_{\mathrm{LF}}^{2}\right) d x^{\prime} d y^{\prime}$. Thus, we can separate $F$ into two contributions, accounting for the LF field $\mathbf{E}_{\mathrm{LF}}$ and for the beam field $\mathbf{E}_{\mathrm{opt}}$, denoting them $F_{\mathrm{el}}$ and $F_{\mathrm{opt}}$, respectively. Writing $I_{\mathrm{opt}}=\frac{2 P}{\pi w^{2}} \exp \left[-\frac{2\left(x^{2}+y^{2}\right)}{w^{2}}\right]$, corresponding to a Gaussian beam of waist $w$ and power $P$, we get:

$$
\begin{aligned}
F_{\mathrm{opt}}(P, w, h) & =\frac{2 \gamma_{\mathrm{opt}} P}{\pi w^{2}} \sum_{l=1}^{\infty} \frac{\sin \left(\frac{\pi l}{2}\right)}{\pi l} \Omega_{l}(y=0 ; h, w), \\
F_{\mathrm{el}}(V) & =\frac{4 \gamma_{\mathrm{LF}} V^{2} S_{1}}{\pi^{3}}
\end{aligned}
$$

where we introduced $S_{1}=\sum_{l=0}^{\infty} \frac{\sin \left[\frac{\pi}{2}(2 l+1)\right]}{[\pi(2 l+1)]^{3}} \approx 0.9689$ [see the Appendix A for details and the definition of $\left.\Omega_{l}(y ; h, w)\right]$. The differences between $F_{\text {opt }}$ and $F_{\text {el }}$ stem from the unequal spatial overlaps between the external fields and the medium (i.e., the associated Green function $G$ ). For narrow beams $(w \ll h)$ we have $F_{\text {opt }}=\gamma_{\text {opt }} P S_{2}(w, h)$, where $S_{2}(w, h)=$ $\sum_{l=0}^{\infty} \frac{1}{\pi(2 l+1)} \operatorname{erfc}\left[\frac{\pi(2 l+1) w}{2 \sqrt{2} h}\right][60]$.

From Eq. (5) it is possible to describe the mechanism behind the FT. We first assume $\partial F / \partial \theta_{m}=0$, corresponding to $n_{e}\left(\theta_{m}\right) \approx n_{\perp}$ in the expression providing $\gamma_{\text {opt }}$. Then, a nontrivial solution beyond $\theta_{m}=0$ exists if

$$
F\left(I_{\mathrm{opt}}, \theta_{m}, V, h\right)>0.5 .
$$

Equation (8) yields the minimum external (pair of) excitation(s) capable to overcome the FT [see Fig. 2(a)]. Under the condition $\partial F / \partial \theta_{m}=0$, it is $\theta_{m}=0^{\circ}$ at the FT, corresponding to a continuous reorientation $\theta_{m}$ versus external stimuli: the phase transition is second-order and hysteresis is inhibited. Fig. 2(b) shows the case when the full expression of $F$ is accounted for. For anisotropies corresponding to standard liquid crystals, there are only minor adjustments to 
(a)

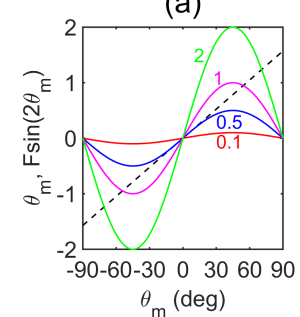

(b)

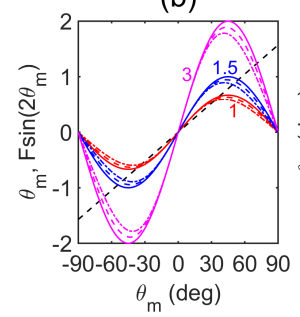

Fig. 2. (Color online) (a) Graphic solution of Eq. (8) parametrized with $F$ (values next to each line). (b) Solution for an optical beam, parametrized with $n_{\perp} F$ (as marked next to each line). Solid lines correspond to $n_{e}\left(\theta_{m}\right) \approx n_{\perp}$, dashed and dash-dotted lines correspond to $\partial F / \theta_{m} \neq 0$ for $n_{\|}=1.7$ and $n_{\|}=2$, respectively. (c) Maximum reorientation $\theta_{m}$ corresponding to the curves plotted in (b) versus magnitude of the external excitation $F$ for $n_{e} \approx n_{\perp}$ (blue), $n_{\|}=1.7$ (black) and $n_{\|}=2$ (red), from top to bottom, respectively. In all plots $n_{\perp}=1.5$.
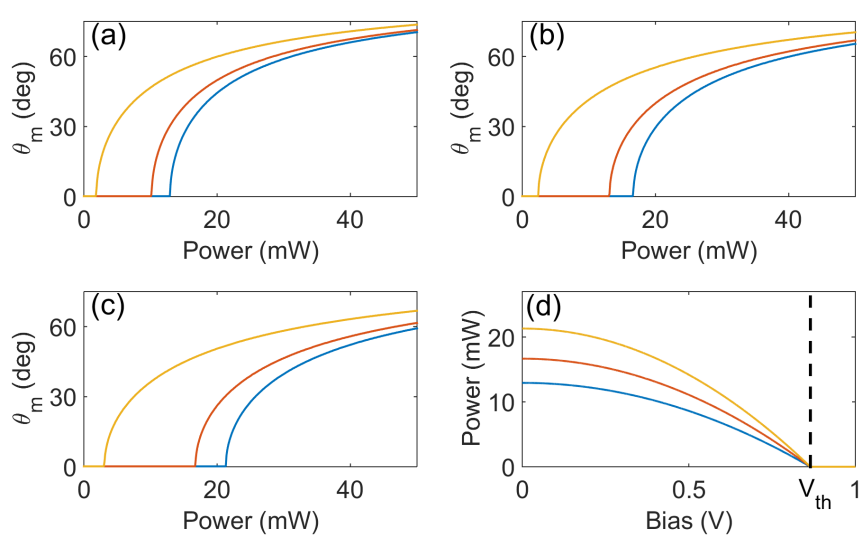

Fig. 3. (Color online) Maximum reorientation angle $\theta_{m}$ versus beam power for (a) $w=2 \mu \mathrm{m}$, (b) $w=5 \mu \mathrm{m}$ and (c) $w=10 \mu \mathrm{m}$, respectively, computed via the semi-analytic approach Eq. (5). The applied bias is $0,0.6$ and $0.8 \mathrm{~V}$ from right to left, respectively. (d) Threshold power $P_{t h}$ versus applied bias $V$ for $w=2,5$ and $10 \mu \mathrm{m}$, from bottom to top, respectively. Here we refer to the mixture E7 at room temperature and a beam wavelength of $1064 \mathrm{~nm}$.

the approximation $n_{e}\left(\theta_{m}\right) \approx n_{\perp}$. Moreover for small $\theta$, i.e., close to threshold, $\partial^{2} F / \partial \theta_{m}^{2}<0$ thus the reorientation curve $\theta_{m}$ versus external excitation $F$ is continuous, confirming that the transition is second-order [38]. Both in the approximate and in the exact cases, given the system symmetry with respect to positive or negative $\theta$, at the FT a pitchfork bifurcation occurs [see Fig. 2(c)] [39], [61].

To compute the optical and electric FT we can use Eq. (5) and consider the two fields applied separately, under the condition $F=0.5$. In the absence of light, Eq. (7) provides the voltage threshold $V_{t h}^{(0)}=\frac{\pi}{2} \sqrt{\frac{\pi K}{\epsilon_{0} \Delta \epsilon_{\mathrm{LF}} S_{1}}}$. The analytical (exact) calculation provides $V_{t h}^{\text {exact }}=\pi \sqrt{\frac{K}{\epsilon_{0} \Delta \epsilon_{\mathrm{LF}}}}$ [39], [40], thus $V_{t h}^{(0)} / V_{t h}^{\text {exact }}=0.5 \sqrt{\frac{\pi}{S_{1}}} \approx 0.9$. Conversely, in the absence of voltage, from Eq. (6) the threshold beam power is $P_{t h}^{(0)}=\frac{1}{2 \gamma_{\mathrm{opt}} S_{2}(w, h)}$ [60].

When both light and voltage are applied simultaneously, the power threshold $P_{t h}$ for each value of the applied bias $V$ can be found by equating the left hand side of Eq. (8) to 0.5. Using Eqs. (6-7), the threshold beam power $P_{t h}$ for narrow beams

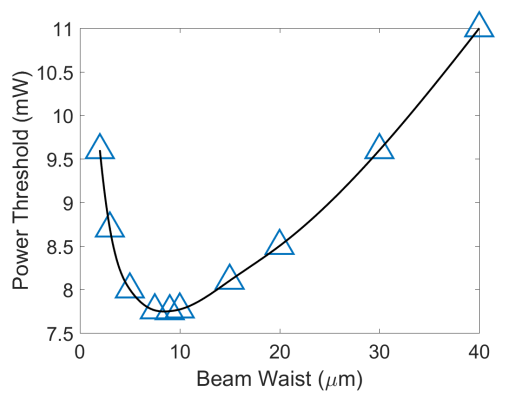

Fig. 4. Beam power threshold versus input waist in a cylindrical geometry with a radius of $400 \mu \mathrm{m}$ and propagation length $L_{z}=1.5 \mathrm{~mm}$. Triangles result from numerical simulations, the solid line is the spline interpolation of the numerical data.

is given by:

$$
P_{t h}(V ; w, h)=\left[1-\left(\frac{V}{V_{\mathrm{th}}^{(0)}}\right)^{2}\right] P_{t h}^{(0)} .
$$

Consistently with the experiments described in the second part of this Paper, hereafter we focus on the specific NLC mixture E7 in a planar cell of thickness $h=100 \mu \mathrm{m}$, excited by a laser beam at $\lambda=1064 \mathrm{~nm}$. The relative dielectric constants for the quasi-static LF field are 19.6 and 5.1, for fields parallel and normal to $\mathbf{n}$, respectively. The refractive indices $n_{\perp}$ and $n_{\|}$are 1.5038 and 1.6954 at room temperature, respectively. The single Frank elastic constant (taken equal for splay, bend and twist deformations) is $12 \times 10^{-12} \mathrm{~N}$. Fig. 3 shows the maximum angle $\theta_{m}$ versus input power $P$ computed via Eq. (5) for three beam sizes [Fig. 3(a-c)]. At a fixed power the reorientation increases for narrower beams owing to the stronger field $\mathbf{E}_{\text {opt }}$, while the reorientation curves shift to the left (i.e., less power is required to reach the same $\theta_{m}$ ) as the bias increases. For $V>V_{t h}^{(0)}$, the voltage overcomes the (electric) FT and reorientation is finite even without light power $P$. The comparison with the full numerical solution developed and shown in ref.[36] and supplemental material shows a good match between the two approaches, with the accuracy of the analytic approach decreasing as the beam width increases, consistently with the assumptions. Finally, Fig. 3(d) graphs the optical threshold power $P_{t h}$ versus applied voltage [Eq. (9)] for three beam widths. The trend is parabolic and all curves cross in $P_{t h}=0$ when $V=V_{t h}^{(0)}$.

2) Longitudinal effects: Although the applied bias and its effects are essentially invariant versus $z$, in the linear regime the intensity distribution of the beam, $I_{\text {opt }}$, changes in propagation through diffraction. The diffractive spreading is relevant when the Rayleigh distance is comparable with or shorter than the cell length along $z$. Eq. (5) remains valid, but the function $F$ cannot be expressed in a closed form due to a $z$-dependent Green function [57]. Nevertheless, the problem can be addressed numerically. For the sake of simplicity, we assume a cylindrically symmetric sample as the qualitative behavior does not vary with the geometry. Specifically, we solved Eq. (1) setting $E_{\mathrm{LF}}=0$ (unbiased cell) and $I_{\mathrm{opt}}=\frac{2 P}{\pi w^{2}(z)} \exp \left[-\frac{x^{2}+y^{2}}{w^{2}(z)}\right]$, where, consistently with 
free diffraction, $w(z)=w_{0} \sqrt{1+\left(\frac{z}{L}\right)^{2}}$; here $L$ is the Rayleigh distance. In essence, we compute the maximum reorientation angle in the cell versus the input power $P$, the latter curves providing the threshold power for a given input waist $w_{0}$. The threshold power versus beam waist $w_{0}$ (in $z=0$ ) is plotted in Fig. 4 and shows a relative minimum for $w_{0} \approx 8 \mu \mathrm{m}$. In fact, despite the higher intensities in the early propagation stages, narrower beams undergo larger diffraction and, over $L_{z}$, give rise to a weaker light-matter interaction. Conversely, for larger waists the relative weights of the two effects reverse and the lower beam intensity determines higher thresholds.

\section{Role of self-focusing}

In Sec. II-B, we showed that the Fréedericksz transition from the initial distribution to a reoriented state is secondorder, thus hysteresis is not allowed: the dynamics of the system does not depend on its history. The transition can become first-order when the optical nonlinearity is taken into account, as in this regime the beam can change the dielectric properties of the medium [Eq. (1)], in turn modifying its intensity through self-focusing, as modelled by Eq. (2) [62], [63], [64]. In other words, the nonlinearity provides an inherent feedback mechanism, necessary to obtain hysteresis.

The inclusion of feedback in the model developed in Sec. II-B is not straightforward. The actual dynamics, in fact, comprises a Gaussian beam subject to quasi-sinusoidal oscillations in width $w(z)$, the so called breathing ${ }^{2}$. The latter would require computing the Green function $G$ in the full three-dimensional case. Nevertheless, the basic physics can be grasped by assuming a $z$-invariant beam profile: in the equivalent twodimensional model the breathing can be replaced by an average soliton width, corresponding to the shape-preserving solution. In the highly nonlocal limit, the perturbation of the orientation $\theta$ can be approximated by a parabola as in the "accessible soliton" model introduced by Snyder and Mitchell [65], [66], to be slightly amended/adjusted in actual diffusive media [67], [68]. After setting $\theta=\theta_{m}-\theta_{2}\left(x^{2}+y^{2}\right)$, such modified Snyder-Mitchell model provides the implicit equation (see Appendix B)

$$
w_{s}\left(P, V, \theta_{m}\right)=\left(\frac{2 \eta}{n_{e}\left(\theta_{m}\right) n_{e}^{\prime}\left(\theta_{m}\right) k_{0}^{2} P \theta_{2}\left(\theta_{m}, w_{s}, P, V\right)}\right)^{\frac{1}{4}}
$$

where $n_{e}^{\prime}\left(\theta_{m}\right) \equiv \frac{d n_{e}}{d \theta}$ and $\theta_{2}$ is provided by Eq. (14). The solution of (10) yields Eq. (15) in Appendix B. Although the average beam width depends on the input width $w_{0}$, as well [69], this does not alter the basic physics and affects only slightly our results; hence, it will be neglected. Eq. (10) states that the width of the shape-preserving soliton depends on the input power $P$, the bias $V$ and the maximum reorientation $\theta_{m}$, the latter being related to the size of the nonlinearity.

When self-focusing is accounted for, the beam width depends on the input power $P$, with $\theta_{m}$ and the bias $V$ acting as free parameters related to the material response. It is then possible to solve Eq. (5) jointly with Eq. (10) to get the

\footnotetext{
${ }^{2}$ This corresponds to the highly nonlocal limit, that is, when $w \ll h$.
}
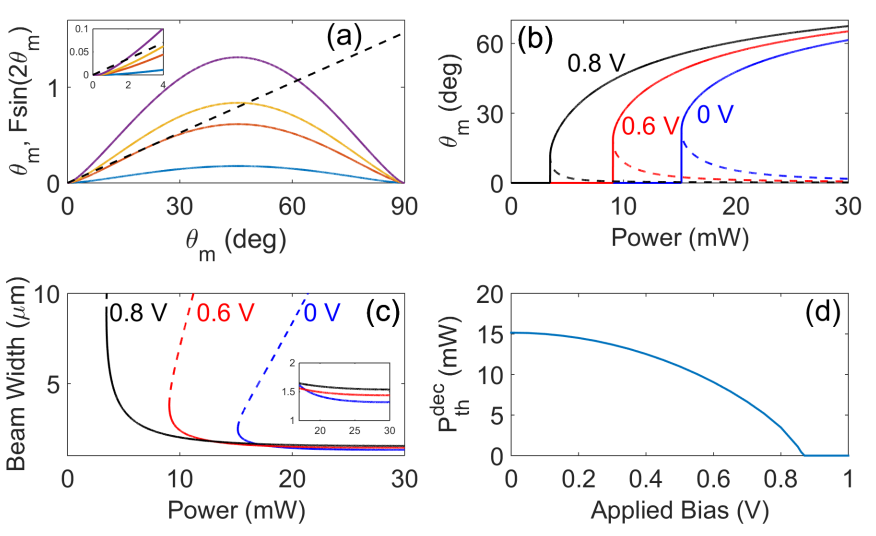

Fig. 5. Reorientation in the presence of self-focusing. (a) Graphic solution of Eq. (5) when (10) is used and the cell is unbiased. From bottom to top, the curves correspond to powers of 5, 15.15 (corresponding to $P_{\mathrm{th}}^{\mathrm{dec}}$ ), 20 and $30 \mathrm{~mW}$, respectively. Inset: zoom around the origin.(b) Maximum reorientation $\theta_{m}$ and (c) beam width versus beam power for three biases, as labelled. Solid and dashed lines correspond to stable and unstable branches, respectively. Inset: zoom at large powers. (d) Dependence of the Fréedericksz threshold $P_{\mathrm{th}}^{\mathrm{dec}}$ from applied voltage. Here we considered the NLC mixture E7 at room temperature and a wavelength of $1064 \mathrm{~nm}$.

reorientation curve versus power in the presence of selftrapping. As sketched in Fig. 5(a) for the unbiased case (the behavior remains similar when a voltage is applied), the function $F$ defined by Eq. (6) does no longer exhibit a sinusoidal trend with respect to $\theta_{m}$. At low powers there are no solutions of Eq. (5) (besides the trivial $\theta_{m}=0$ ) as reorientation does not occur. The Fréedericksz threshold, $P=P_{\text {th }}^{\text {dec }}$ (we introduce the subscript "dec" as this value corresponds to the FT for decreasing powers in the hysteresis loop, see below) is achieved when the two curves are tangent to one another for $\theta_{m} \neq 0$ : reorientation versus input power shows a sudden jump, that is, a first-order transition. For powers $P>P_{\text {th }}^{\text {dec }}$ two solutions can exist [Fig. 5(b-c)] [60]. One family of such solutions exhibits a maximum reorientation $\theta_{m}$ increasing with power [solid lines in Fig. 5(b-c)], and it corresponds to stable solutions (see Appendix C). Vice versa, the other family presents an inverted trend for reorientation versus power [dashed lines in Fig. 5(b-c)], corresponding to unstable solutions (see Appendix C). Maximum reorientation $\theta_{m}$ and beam width are plotted in Fig. 5(b) and Fig. 5(c), respectively. Fig. 5(d) finally illustrates how the Fréedericksz threshold power $P_{\text {th }}^{\text {dec }}$ monotonically decreases with applied bias, going to zero for $V=V_{\mathrm{th}}$.

Optical bistability between diffracting and self-confined beam states is confirmed by direct numerical simulations. For the sake of clarity (and physical intuition) we refer to an unbiased cell: the role and effects of an applied voltage are discussed in Ref. [36].

We start from the solution of the reorientational equation [Eq. (1)] and a constant intensity profile, taken invariant along $z$. The absolute value of the maximum reorientation $\theta_{m}$ is graphed in Fig. 6(a) versus beam power $P$ : the same trend of Fig. 3 is observed.

Next we account for self-focusing by solving the optical evolution equation together with the reorientational equation. 

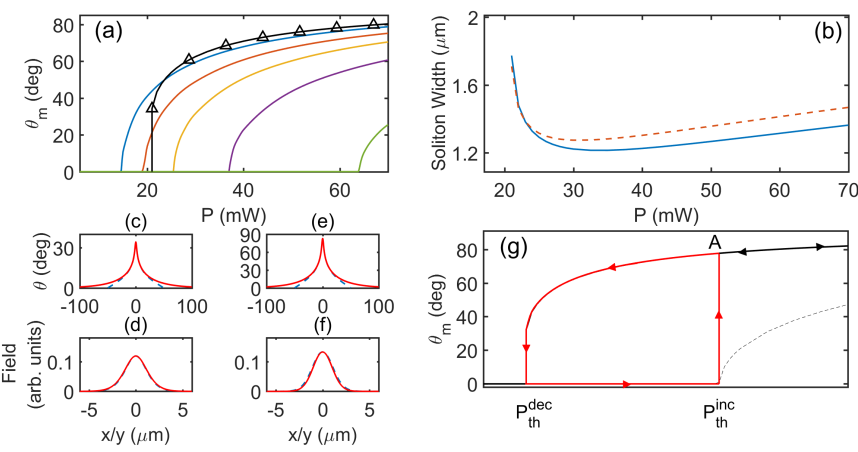

Fig. 6. Bistability in the presence of optical solitons in a planar cell. (a) Maximum reorientation versus input beam power when self-focusing is neglected (beam widths are 2, 5, 10, 20 and $40 \mu \mathrm{m}$, solid lines from blue to red, yellow, violet and green, respectively, from left to right) but feedback on the wavepacket profile is accounted for (black line with triangles). (b) Soliton width versus power along $x$ (dashed line) and $y$ (solid line). Reorientation angle $\theta(\mathrm{c}, \mathrm{e})$ and field profile $(\mathrm{d}, \mathrm{f})$ versus $x$ (dashed blue lines) and $y$ (solid red lines), for $P=20 \mathrm{~mW}$ (c,d) and $P=88 \mathrm{~mW}$ (e,f). (g) Bistable loop in the plane $\theta_{m}$-power between diffracting (portion where $\theta_{m}$ vanishes) and self-confined states (finite $\theta_{m}$ ).

We look for $z$-invariant solitary wavepackets with the ansatz $A=u_{s}(x, y) e^{i k_{0} n_{s} z}$ and $\theta=\theta_{s}(x, y)$. The substitution leads to a nonlinear eigenvalue problem, the solutions of which are plotted in Fig. 6(b-f). In agreement with Fig. 5, solitons do not exist for powers below a threshold, $P_{\mathrm{th}}^{\mathrm{dec}}$, owing to the Fréedericksz transition. The reorientation curve in the presence of self-focusing is plotted in Fig. 6(a) (black line with triangles): for $P<P_{\text {th }}^{\text {dec }}$, self-focusing does not occur and the beam diffracts. Within the soliton existence interval, $u_{s}$ is Gaussian within a good accuracy, and $\theta_{s}$ maintains the same spatial profile regardless of the input power. Specifically, the nonlinear optical perturbation is parabolic near the beam axis and close to the Green function away from it [Fig. 6(cf)]. We stress that both beam profile and molecular distribution are approximately circularly symmetric. Fig. 6(g) shows the hysteresis loop corresponding to the first-order transition. For a given input field (Gaussian beam of waist $w_{0}$ and planar phase front in $z=0$ ), we gradually increase the input power from zero: the wavepacket diffracts until $P=P_{\mathrm{th}}^{\text {inc }}$, where reorientation at FT occurs. The minimum power $P_{\text {th }}^{\text {inc }}$ to form a solitary wave depends on the initial width of the beam $w_{0}$, see Fig. 3 and Fig. 4. For further power increases, self-focusing comes into play and, consequently, the beam profile changes towards a self-confined state. We pinpoint once again that, in actual samples, a breather soliton is normally excited, with width oscillating around the shape-preserving solution shown in Fig. 6(b). In any event, the reorientational curve differs from the initial one, so that when the beam power is ramped down, self-trapping is maintained until the soliton exists, i.e., down to the (lower) threshold $P=P_{\mathrm{th}}^{\mathrm{dec}}$.

\section{EXPERIMENTAL RESULTS}

\section{A. Bistability versus input beam power}

From the results shown in Fig. 3(d), one expects the wider hysteresis loops to occur in unbiased cells. However, for $V=0$, the power corresponding to optical FT is relatively high and spatial solitons tend to be temporally unstable, with path fluctuations [70], [62]. The application of a low-frequency electric field $\mathbf{E}_{\mathrm{LF}}$ can reduce the power threshold $P_{\mathrm{th}}^{\text {inc }}$ [see Fig. 3] and make the solitons observable [63] as FT is reached by the combined action of voltage and beam induced torques. We first measured the beam width as a function of the applied voltage at fixed power [64]. Fig. 7(a) shows the average beam width versus bias for $P=2 \mathrm{~mW}$. The beam width was obtained from the intensity profile in the plane $y z$, acquired from the light scattered out of the plane (see Appendix D), through a best-fit procedure with a fundamental Gaussian. The average width was calculated as $w_{z}=\frac{1}{L_{z}} \int_{0}^{L_{z}} w(z) d z$, with $L_{z}$ the length of the NLC cell along $z$. An abrupt drop in $w_{z}$ is observed when $V_{\mathrm{th}} \approx 1.1 \mathrm{~V}$, where the molecules start to rotate. At $V \approx 1.5 \mathrm{~V}$, the beam width $w_{z}$ reduces to a value only slightly wider than the input, i.e., a self-trapped beam is formed. Fig. 7(b) shows the corresponding intensity evolution in the plane $y z$ and the output profile in $x y$. When the beam width starts to reduce $(0.9 \mathrm{~V}<V<1.1 \mathrm{~V})$, the output profile (bottom photographs) shows a wide profile across $y$ and fringes across $x$ : the molecules are reoriented by the bias, leading to a $y$-invariant multi-mode waveguide, without an appreciable role of all-optical reorientation. For $1.1 \mathrm{~V}<V<1.3 \mathrm{~V}$, i.e., corresponding to the drop in Fig. 7(a), the waveguide becomes more confining owing to the larger $\theta_{m}$, supporting a light stripe parallel to $y$ at the output. As $V$ is further increased, nonlinear confinement occurs across $y$ (already appreciable for $V=1.3 \mathrm{~V}$ ). Finally, at $V=1.5 \mathrm{~V}$, a cylindrically-symmetric self-confined beam is observed, a nematicon invariant in $y z$ and transversely confined in $x y$ [63]. A nematicon appears only for large enough voltages owing to the modulation of the nonlinearity with the initial orientation angle $\theta$ in the absence of light [57]. The $x$-shift of the output beam vs voltage is due to the walk-off angle varying with $\theta$ [71], [72].

According to our model, bistability is not expected for $V>$ $V_{\text {th }}$; this was confirmed by measuring the beam width versus the input power for $V=1 \mathrm{~V}$ (see Fig. 7(b)) [63], i.e., just above the FT. The intensity profiles are the same for increasing vs decreasing powers. Furthermore, stable self-localized beams exist for $P \approx 12 \mathrm{~mW}$ : the power threshold is lowered by the joint effect of light and bias, in agreement with Fig. 3.

Therefore a trade-off needs to meet to obtain bistability. On the one hand, it must be $V<V_{\mathrm{th}}$ to ensure the existence of the diffracting state when light power increases from zero. On the other hand, too low biases correspond to unstable solitons because of the large power required to support self-guidance. With reference to Fig. 3(d), the width of the hysteresis loop decreases and eventually vanishes as $V$ approaches $V_{\mathrm{th}}$ : in fact, the loop size is determined by the separation between reorientation curves corresponding to different beam widths. With these considerations in mind, we measured the hysteresis loop for $V=0.92 \mathrm{~V}$, just below the FT, near room temperature $T=18^{\circ} \mathrm{C}$. The experimental results are shown in Fig. 8, with photographs of beam propagation in $y z$ and output profiles in $x y$, as well as a graph the average beam width versus power. For powers up to $P=13 \mathrm{~mW}$, the beam propagated 
(a)

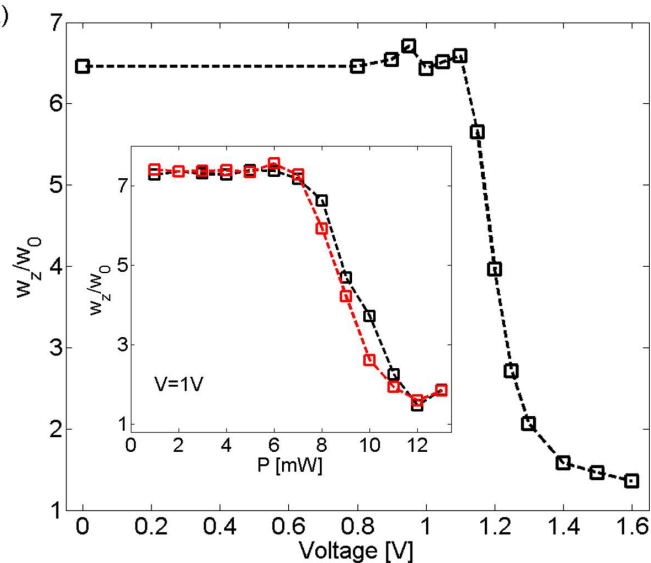

(b)
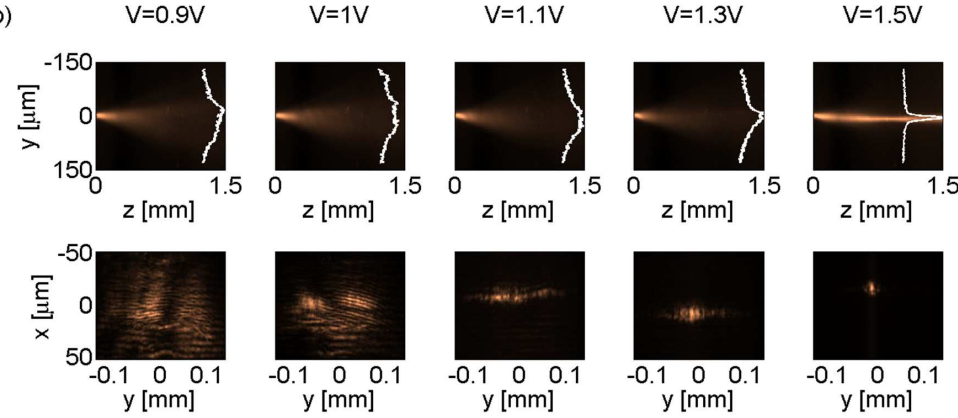

y $[\mathrm{mm}]$ $\mathrm{y}[\mathrm{mm}]$

Fig. 7. (a) Electric FT (EFT) for a $P=2 \mathrm{~mW}$ beam as calculated from the acquired propagation in $y z$. The inset shows the average beam width along $z$ versus input power, for $V=1.1 \mathrm{~V}$ corresponding to the electric FT threshold, for increasing (black squares and line) and decreasing (red squares and line) powers: no hysteresis is observed. (b) Photographs of (top) beam propagation in $y z$ and (bottom) output profiles in $x y$ for several voltages. The voltage driven reorientation occurs sooner across $x$, with the beam confined in the linear index well. The actual threshold value is lower than derived from (a).

linearly with no reorientation. Then the beam size started to reduce without reaching a self-trapped state. A self-confined state could be observed at $P=16.5 \mathrm{~mW}$ (corresponding to $\left.P_{\mathrm{th}}^{\text {inc }}\right)$, when the beam width suddenly collapsed. The power was then raised up to $P \approx 20 \mathrm{~mW}$, where stable solitons could still be observed. To complete the counterclockwise hysteresis loop the beam power was decreased back and the self-confined beam survived down to $P=14 \mathrm{~mW}$ (corresponding to $P_{\mathrm{th}}^{\mathrm{dec}}$ ); for further reductions a diffracting state was retrieved. Fig. 8(e) shows the measured hysteresis, with a loop width of about $3 \mathrm{~mW}$.

1) Temperature dependence: A finite FT derives from the unavoidable fluctuations associated with a finite temperature [39], [51]. The noise-induced rotation of an ensemble of molecules gets amplified by the external field up to macroscopic scales and transferred to the surrounding molecules via elastic interactions, provided the torque is strong enough. Hence, temperature is expected to play a central role. A control of the sample temperature allows optimizing and tuning the occurrence and the size of the hysteresis loop. High temperatures increase the noise contribution to the system energy, flattening the potential barrier and preventing bistability; low temperatures reduce the nonlinearity, preventing efficient self-confinement and increasing the relaxation time in the transitions between the two states. We repeated the measurements shown in Fig. 8 at $T=16^{\circ} \mathrm{C}$ and $T=23^{\circ} \mathrm{C}$, see Fig. 9(a-b) [73]. The higher temperature lowered both the power thresholds $P_{\mathrm{th}}^{\mathrm{inc}}$ and $P_{\mathrm{th}}^{\mathrm{dec}}$ due to stronger fluctuations; the loop size, $P_{\mathrm{th}}^{\mathrm{inc}}-P_{\mathrm{th}}^{\mathrm{dec}}$, shrunk as well. These results are in qualitative agreement with the model [Fig. 9(c)] accounting for the three different values of the elastic constants associated with splay, bend and twist deformations [39] (see the Supplemental Material in Ref. [36] for more details). The temperature dependence of the elastic constant and the refractive indices are taken into account [74], [75].

\section{B. Optical bistability versus applied voltage}

Equation (1) states that reorientation can be triggered by low- and optical frequency fields and Eq. (9) describes the FT threshold in terms of their joint action. Hence, we should be able to observe optical bistability when varying the applied voltage while keeping the input beam conditions constant. As shown in Fig. 7(a), we investigated the propagation of a $P=2 \mathrm{~mW}$ beam for various applied voltages: selfconfinement occurred when all-optical and electric responses sufficed to overcome the FT [37]. This was observed above $V=1 \mathrm{~V}$, when the beam width gradually reduced until it resulted into a spatial soliton. However, the transition between diffracting and self-confined states was smooth, preventing the observation of bistability: the curves for increasing and decreasing biases superposed and no hysteresis could be observed. As apparent from the simulations in Fig. 10, in order to obtain self-confinement for low beam powers, the voltage must be high enough to overcome the FT on its own, which implies a second-order transition. Conversely, large enough beam powers make $V_{t h}$ appreciably dependent on beam width, paving the way to a first-order transition.

Experimentally, we varied the relative weights of the two reorientational torques. First, we observed a reduction of the voltage needed to overcome the FT as the optic power increased, as in Fig. 11(a) in good agreement with Fig. 3(d). In Fig. 11(b) it is apparent that higher powers correspond to steeper transitions versus applied bias, with an abrupt switch (the slope approaches $\pi / 2$ ) between diffracting and self-trapped states - a first-order transition - for $P=25 \mathrm{~mW}$. Higher powers would allow the light beam to overcome the FT by itself when self-trapped [Fig. 3(d)].

Figure 12 shows the beam evolution for $P=25 \mathrm{~mW}$. As the bias increases, the electric FT occurred for $V \geq 0.88 \mathrm{~V}$ : above this value, the beam turns into a self-confined wave, with a sharp transition [Fig. 12(e)]. A narrower beam yields a stronger light-matter interaction even when decreasing the 
(a)

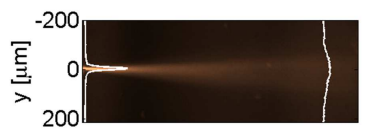

(b)
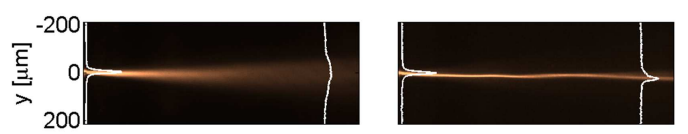

(c)
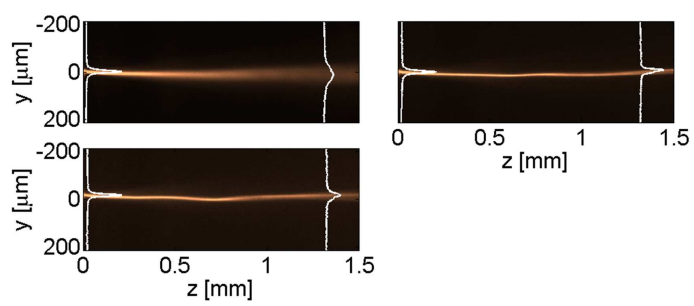

(d)

(e)

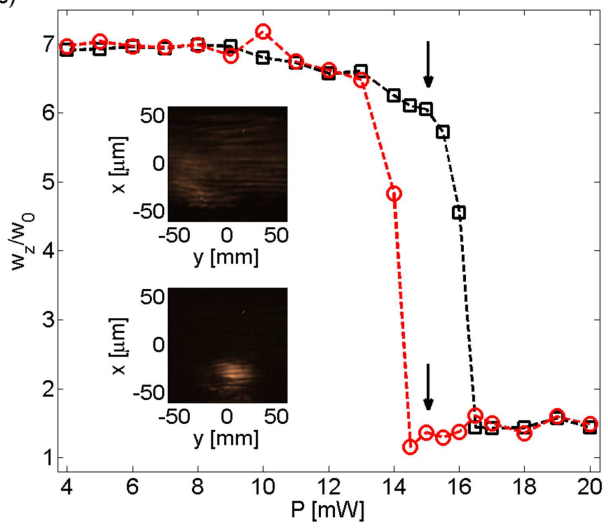

Fig. 8. Bistable beam propagation versus input beam power for $V=0.92 \mathrm{~V}$. (a)At $P=1 \mathrm{~mW}$ the beam diffracts, so it does for (b)-left $P=15.5 \mathrm{~mW}$ and (c)-left $P=16.5 \mathrm{~mW}$. When the FT is overcome, a nematicon forms, as in (d) for $P=20 \mathrm{~mW}$. When decreasing the input power after reaching the self-confined state, the narrower beam corresponds to a lower threshold

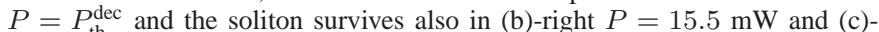
right $P=16.5 \mathrm{~mW}$. (e) Hysteresis of the average beam width, for increasing (black squares) and decreasing (red circles) input power. The insets show the output intensity profiles at the points indicated by the arrows $(P=16.5 \mathrm{~mW})$, in either the diffracting (top) or the self-confined (bottom) states.
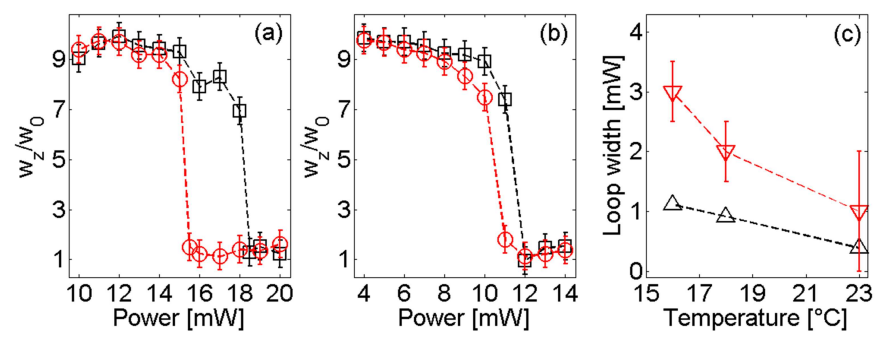

Fig. 9. Temperature dependence of the hysteresis loop. Average beam width for $T=16^{\circ}$ (a) and $23^{\circ} \mathrm{C}$ (b) for increasing (black squares) and decreasing (red circles) input power. (c) Measured (black triangles) and calculated (red inverted triangles) loop width versus temperature.
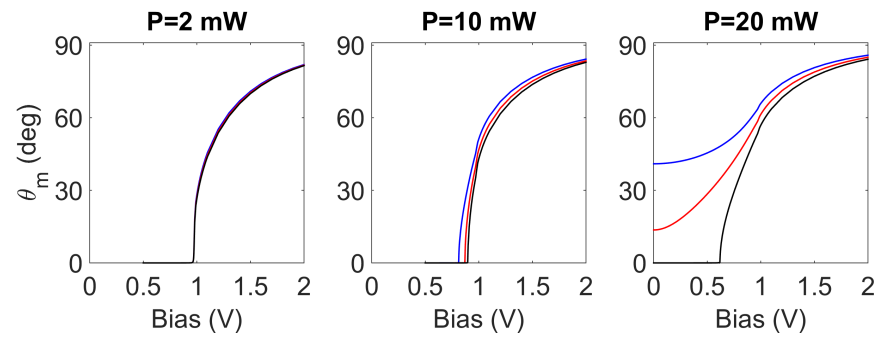

Fig. 10. Maximum reorientation angle $\theta_{m}$ versus applied bias for three beam powers, computed by solving numerically Eq. (1). In each panel the beam width is $2 \mu \mathrm{m}$ (blue line), $5 \mu \mathrm{m}$ (red line) and $10 \mu \mathrm{m}$ (black line), from left to right, respectively. (a)

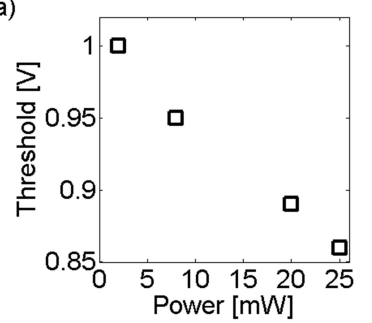

(b)

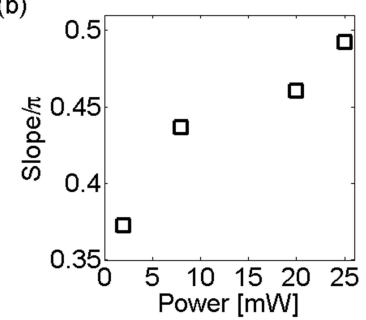

Fig. 11. (a) Threshold voltage $V_{t h}$ and (b) transition slope of the loop vs bias graphed as a function of the input beam power, the latter kept constant during each cycle. For $P=25 \mathrm{~mW}$ the transition slope is large enough to be considered first-order and support bistability.

bias, with reorientational solitons surviving in a wider interval $0.84 \mathrm{~V} \leq V \leq 0.88 \mathrm{~V}$ and diffraction occurring only at lower voltages than in the ramp-up branch. Similar to the power-driven hysteresis discussed in Sec. III-A, all the states in the loop are stable, demonstrating that bistability stems from the interacting fields and the feedback provided by the reorientational response, regardless of the specific optical or electric mechanism.

\section{CONCLUSiOnS}

We reported nonlinear beam propagation in nematic liquid crystals in the presence of Fréedericksz threshold, describing the propagation of light wavepackets in planar cells versus input optical power and applied voltage. For propagation distances exceeding the Rayleigh length, the feedback inherent to self-focusing via reorientation transforms a second-order into a first-order transition, resulting in bistability and hysteresis between diffracting and self-confined beam states. The basic ingredient is the dependence of the power threshold at the Fréedericksz transistion on the beam profile: a self-trapped beams provides larger light-matter coupling, in turn lowering the threshold.

We demonstrated optical bistability using either the input beam power or the applied voltage as control parameters. The occurrence of bistability strongly depends on the joint action of all-optical and electro-optic responses. For power-control, if the bias is above the Fréedericksz threshold, bistability is inhibited. For biases below threshold, the lower the voltage the wider the hysteresis loop, with a marked dependence on temperature, as well. In experiments, a minimum bias was 
(a)

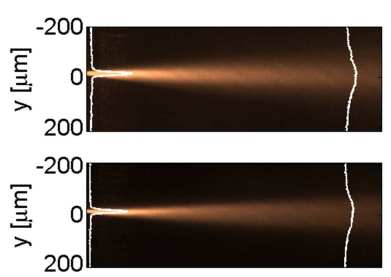

(c)
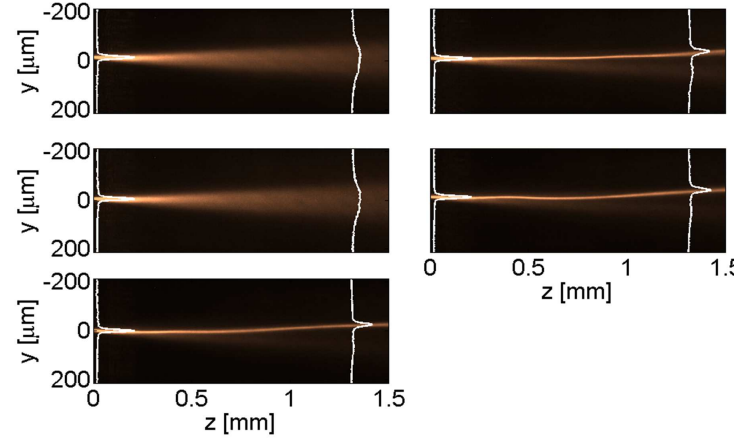

(e)

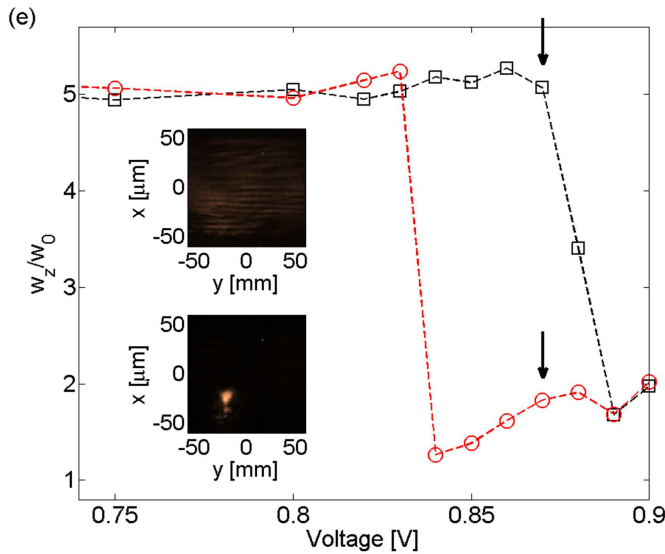

Fig. 12. Bistable beam propagation versus voltage, for $P=25 \mathrm{~mW}$. At (a) $V=0.70 \mathrm{~V}$ the beam diffracts, as for (b)-left $V=0.84 \mathrm{~V}$ and (c)-left $V=0.85 \mathrm{~V}$. (d) When the FT is overcome, a nematicon forms for $V=$ $0.90 \mathrm{~V}$. Decreasing the voltage after the self-confined state has been reached, the narrower beam lowers the threshold values and the soliton survives also for (b)-right $V=0.84 \mathrm{~V}$ and (c)-right $V=0.85 \mathrm{~V}$. (e) Hysteresis of average beam width vs bias, for increasing (black squares and line) and decreasing (red circles and line) voltages. The insets show the output intensity profiles in $x y$ corresponding to the points indicated by the arrows $(V=0.87 \mathrm{~V})$, in the diffracting (top) and self-confined (bottom) states. Here the temperature was $T=19^{\circ} \mathrm{C}$

required to avoid beam instabilities, with a measured hysteresis loop width of about $3 \mathrm{~mW}$. For voltage control, hysteresis can be achieved by ramping the bias at fixed beam power. The requirement on input power is twofold: it cannot be too low to provide a nonlinear response and must ensure that reorientation vanishes for a finite voltage in the presence of a self-trapped beam. We observed a maximum loop size of $4 \mathrm{mV}$ for $\mathrm{P}=25 \mathrm{~mW}$. The demonstration of optical bistability with propagating beams in nematic liquid crystals can be foreseen in other reorientational media [76], [77] and paves the way to a novel class of sequential logics and memory elements based on light self-confinement.
APPENDIX A

SOlution OF THE POISSON EQUATION FOR A GAUSSIAN INPUT

The generic solution of the standard Poisson equation in two dimensions $\frac{\partial^{2} g}{\partial x^{2}}+\frac{\partial^{2} g}{\partial y^{2}}=\kappa f(x, y)$ is obtained via the superposition principle $g(x, y)=$ $\kappa \int_{-\infty}^{\infty} \int_{-h / 2}^{h / 2} f\left(x^{\prime}, y^{\prime}\right) G\left(x, y, x^{\prime}, y^{\prime}\right) d x^{\prime} d y^{\prime}$, with the Green function $G$ expressed by (4) in our geometry. For a Gaussian input $f(x, y)=\exp \left[-2\left(x^{2}+y^{2}\right) / w^{2}\right]$, we find [59]

$$
g=-\kappa \sum_{l=1}^{\infty} \frac{1}{\pi l} \Omega_{l}(y ; h, w) \sin \left[\frac{\pi l(x-h / 2)}{h}\right] .
$$

In Eq. (11) we introduced the $y$-dependent quantity $\Omega_{l}(y ; h, w)=\Omega_{l}^{x}(h, w) \Omega_{l}^{y}(y ; h, w)$, with

$$
\begin{array}{r}
\Omega_{l}^{x}(h, w)=\int_{-h / 2}^{h / 2} e^{-\frac{2 x^{2}}{w^{2}}} \sin \left[\frac{\pi l(x-h / 2)}{h}\right] d x, \\
\Omega_{l}^{y}(y ; h, w)=\frac{\sqrt{\pi} w}{2 \sqrt{2}} e^{\frac{\pi^{2} w^{2} l^{2}}{8 h^{2}}}[W(y ; h)+W(-y ; h)],
\end{array}
$$

where $W(y ; h) \equiv \operatorname{erfc}[\sqrt{2} y / w+\pi l w /(2 \sqrt{2} h)] e^{\pi l(y / h)}$, with $\operatorname{erfc}(y) \equiv(2 / \sqrt{\pi}) \int_{x}^{\infty} e^{-t^{2}} d t$. In the limit of narrow beams $(w \ll h)$ Eq. (12) provides $\Omega_{m}^{x} \approx$ $(\sqrt{\pi} w / \sqrt{2}) \sin \left(\frac{\pi l}{2}\right) e^{-\frac{\pi^{2} w^{2} l^{2}}{8 h^{2}}}$.

\section{APPENDIX B}

\section{NEMATICONS IN THE HIGHLY NONLOCAL LIMIT}

According to the accessible soliton model [65], [48], the nonlinear index well can be approximated by a parabola. Expanding the reorientational equation (1) around the beam axis (i.e., $x=y=0$ ), for the second derivative of $\theta$ at each beam location we find [66]

$$
\theta_{2}\left(\theta_{m}, w, P\right)=\frac{\sin \left(2 \theta_{m}\right)}{4}\left(\frac{2 \gamma_{\mathrm{opt}} P}{\pi w^{2}}+\frac{\gamma_{\mathrm{LF}} V^{2}}{h^{2}}\right) .
$$

In writing (14) we assumed a cylindrical symmetry for $\theta$ in proximity of the beam, as confirmed with good accuracy by full numerical simulations [see Fig. 6] [36]. The beam width $w_{s}$ of the shape-preserving nematicon provided by Eq. (10) can then be found by using the well known formula for the quantum harmonic oscillator. In fact, making the approximation $\Delta n_{e}^{2} \approx 2 n_{e}^{\prime}\left(\theta_{m}\right) n_{e}\left(\theta_{m}\right)$ in Eq. (2), the soliton width is provided by $w_{s}=\left(\frac{2 \eta}{k_{0}^{2} n_{e}\left(\theta_{m}\right) n_{e}^{\prime}\left(\theta_{m}\right) \theta_{2}}\right)^{1 / 4}$. The corrective factor $\eta=2$ accounts for departure from the original SnyderMitchell model when the nonlinearity is ruled by a Poisson equation, see Refs. [67], [68]. Using Eq. (14) the soliton width $w_{s}$ is

$$
\begin{aligned}
& w_{s}= \sqrt{\frac{\gamma_{\mathrm{opt}} P}{\pi \gamma_{\mathrm{LF}}}} \frac{h}{V} \times \\
& \sqrt{-1+\sqrt{1+\frac{8 \eta \pi^{2} \gamma_{\mathrm{LF}} V^{2}}{h^{2} k_{0}^{2} n_{e}\left(\theta_{m}\right) n_{e}^{\prime}\left(\theta_{m}\right) \sin \left(2 \theta_{m}\right) \gamma_{\mathrm{opt}}^{2} P^{2}}}}
\end{aligned}
$$


In unbiased cells $(V=0)$, Eq. (15) in the limit $V \rightarrow 0$ yields [69]

$$
w_{s}\left(P, \theta_{m}\right)=\frac{1}{k_{0}}\left[\frac{4 \pi \eta}{\gamma_{\mathrm{opt}} \sin \left(2 \theta_{m}\right) n_{e}\left(\theta_{m}\right) n_{e}^{\prime}\left(\theta_{m}\right)} \frac{1}{P}\right]^{1 / 2} .
$$

We finally stress that, with respect to the case $V=0$, the presence of the static field $\mathbf{E}_{\mathrm{LF}}$ introduces a further screening, in turn lowering the effective nonlocality [48], [78].

\section{APPENDIX C}

\section{SOLITON STABILITY IN UNBIASED CELLS}

The stability of the solutions can be derived by considering the free energy $\mathcal{F}$ of the NLC subject to external fields, in particular its dependence on the maximum angle $\theta_{m}$. We refer to the unbiased case for simplicity, as in this limid closed form solutions are available and correspond to Eq. (11). We also neglect the dynamics along $z$, considering invariant beams in propagation. The free energy is the sum of the elastic energy due to NLC distortion and the electromagnetic energy [39]. In the framework of the continuum theory and under the single elastic constant approximation [39], the elastic contribution is $\mathcal{F}_{e l}=\frac{K}{2} \nabla \theta \cdot \nabla \theta$. The electromagnetic term is $\mathcal{F}_{e m}=\frac{1}{2}\left[\epsilon_{0} n_{\perp} \mathbf{E}_{o p t}^{2}+\epsilon_{0} \epsilon_{a}\left(\hat{n} \cdot \mathbf{E}_{o p t}\right)^{2}-\frac{\mathbf{B}_{o p t}^{2}}{\mu_{0}}\right]$, where $\mathbf{B}_{o p t}$ is the magnetic field. By integrating the sum $\mathcal{F}_{e l}+\mathcal{F}_{e m}$ through the cross section, i.e., $F=\int_{-\infty}^{\infty} \int_{-h / 2}^{h / 2}\left(\mathcal{F}_{e l}+\mathcal{F}_{e m}\right) d x d y$, we obtain the system free energy as [73]:

$$
\begin{array}{r}
F \approx \alpha \kappa^{2} \theta_{m}^{2}+\frac{\Gamma \epsilon_{a}}{2 \pi} \frac{P}{n_{s} w^{2}} \int_{-h / 2}^{h / 2} d x \int_{-\infty}^{\infty} \cos (2 \theta) e^{-2 \frac{x^{2}+y^{2}}{w^{2}}} d y+ \\
\Gamma\left[\frac{1}{k_{0}^{2} n_{s} w^{2}}+n_{s}+\frac{\epsilon_{a}}{4} \frac{\cos \left(2 \theta_{m}\right)}{n_{s}}\right] P,
\end{array}
$$

where $\alpha=\frac{1}{4} \sum_{p=0}^{\infty} \sum_{-\infty}^{\infty}\left[\pi^{2} \frac{\Omega_{2 p+1}^{2}}{h}+h\left(\frac{d \Omega_{2 p+1}}{d y}\right)^{2}\right]$, $\kappa(w)=-\frac{1}{\sum_{p=1}^{\infty} \Omega_{p}(0) \sin \left(\frac{\pi p}{2}\right)}$ and $\Gamma=\frac{\epsilon_{0} Z_{0}}{K}$. Below FT, i.e., $P<P_{\text {the }}^{\text {dec }}$, the system exhibits an absolute minimum in $\theta_{m}=0$, in agreement with Fig. 5(a). Above FT, i.e., $P>P_{\text {th }}^{\text {dec }}$, a new local minimum arises for $\theta_{m} \neq 0^{\circ}$. Between the two minima, a local maximum appears. The minimum in $\theta_{m}=0^{\circ}$ is associated to diffracting beams, the other minimum is a stable self-confined wavepacket [solid lines in Fig. 5(bc)] [79]; the local maximum represents a wider and unstable soliton solution [dashed lines in Fig. 5(b-c)].

\section{APPENDIX D \\ SAMPLE GEOMETRY AND SETUP}

The planar cell consists of two glass slides separated by $h \approx$ $100 \mu \mathrm{m}$ using Mylar spacers. Indium Tin Oxide transparent thin film electrodes are deposited on the inner surfaces and allow for the application of the low-frequency electric field, a sinusoidal AC voltage at $1 \mathrm{kHz}$. The inner surfaces were also coated with polyimide polymer, mechanically rubbed to obtain molecular alignment along the $z$ direction (Fig. 1). Two further glass cover slides sealed the regions at input and output facets. Using a microscope objective, the $x$-polarized $\mathrm{TEM}_{00}$ mode of a laser at $\lambda=1064 \mathrm{~nm}$ was focused into the sample to a waist $w_{0} \approx 2 \mu \mathrm{m}$. Out-of-plane scattered light was collected by a CCD camera to acquire the beam evolution in the propagation plane, while a microscope objective imaged the beam output on another CCD device [46].

\section{ACKNOWLEDGEMENTS}

A. A. and G. A. acknowledge the Academy of Finland for support through the Finnish Distinguished Professor grant no. 282858 and the European Union for the COST action IC1208.

\section{REFERENCES}

[1] H. M. Gibbs, Optical Bistability: Controlling Light with Light. San Diego: Academic Press, 1985.

[2] A. Szoke, V. Daneu, J. Goldhar, and N. A. Kurnit, "Bistable optical element and its applications," Appl. Phys. Lett., vol. 15, no. 11, pp. 376379, 1969. [Online]. Available: http://link.aip.org/link/?APL/15/376/1

[3] H. M. Gibbs, S. L. McCall, and T. N. C. Venkatesan, "Differential gain and bistability using a sodium-filled fabry-perot interferometer," Phys. Rev. Lett., vol. 36, pp. 1135-1138, May 1976.

[4] J.-F. Song, X.-S. Luo, A. E.-J. Lim, C. Li, Q. Fang, T.-Y. Liow, L.-X. Jia, X.-G. Tu, Y. Huang, H.-F. Zhou, and G.-Q. Lo, "Integrated photonics with programmable non-volatile memory," Sci. Rep., vol. 6, p. 22616, 2016.

[5] X. J. M. Pang, W. He and P. S. J. Russell, "All-optical bit storage in a fibre laser by optomechanically bound states of solitons," Nature. Phot., vol. 10, pp. 454-458, 2016.

[6] E. Abraham and S. D. Smith, "Optical bistability and related devices," Rep. Prog. Phys., vol. 45, no. 8, p. 815, 1982.

[7] E. Bernabeu, P. M. Mejas, and R. Martnez-Herrer, "Optical bistability: towards all-optical devices," Physica Scripta, vol. 36, no. 2, p. 312, 1987.

[8] D. Fitsios, K. Vyrsokinos, A. Miliou, and N. Pleros, "Memory speed analysis of optical ram and optical flip-flop circuits based on coupled soa-mzi gates," IEEE J. Sel. Top. Quantum Electron., vol. 18, no. 2, pp. 1006-1015, March 2012.

[9] C. Vagionas, D. Fitsios, K. Vyrsokinos, G. T. Kanellos, A. Miliou, and N. Pleros, "Xpm- and xgm-based optical ram memories: Frequency and time domain theoretical analysis," IEEE J. Quantum Electron., vol. 50, no. 8, pp. 1-15, Aug 2014.

[10] X. Fang, K. F. MacDonald, and N. I. Zheludev, "Controlling light with light using coherent metadevices: all-optical transistor, summator and invertor," Light Sci. Appl., vol. 4, p. e292, 2015.

[11] C. Ríos, M. Stegmaier, P. Hosseini, D. Wang, T. Scherer, C. D. Wright, H. Bhaskaran, and W. H. P. Pernice, "Integrated all-photonic non-volatile multi-level memory," Nat. Photon., vol. 9, pp. 725-732, 2015.

[12] Q. Wang, E. T. F. Rogers, B. Gholipour, C.-M. Wang, G. Yuan, J. Teng, and N. I. Zheludev, "Optically recongurable metasurfaces and photonic devices based on phase change materials," Nature Photon., vol. 10, pp. 60-66, 2016.

[13] P. W. Smith, J.-P. Hermann, W. J. Tomlinson, and P. J. Maloney, “Optical bistability at a nonlinear interface," Appl. Phys. Lett., vol. 35, no. 11, pp. 846-848, 1979.

[14] W. Chen and D. L. Mills, "Gap solitons and the nonlinear optical response of superlattices," Phys. Rev. Lett., vol. 58, pp. 160-163, Jan 1987. [Online]. Available: http://link.aps.org/doi/10.1103/PhysRevLett.58.160

[15] D. L. Mills and S. E. Trullinger, "Gap solitons in nonlinear periodic structures," Phys. Rev. B, vol. 36, pp. 947-952, Jul 1987. [Online]. Available: http://link.aps.org/doi/10.1103/PhysRevB.36.947

[16] J. E. Sipe and H. G. Winful, "Nonlinear schroedinger solitons in a periodic structure," Opt. Lett., vol. 13, no. 2, pp. 132-134, 1988.

[17] G. Assanto and G. Stegeman, "Optical bistability in nonlocally nonlinear periodic structures," Appl. Phys. Lett., vol. 56, no. 23, pp. 2285-2287, JUN 41990.

[18] G. Leo, M. Picciau, and G. Assanto, "Guided-wave optical bistability through nonlinear cascading in a phase-matched distributed reflector," Electron. Lett., vol. 31, pp. 1661-1662(1), 1995.

[19] E. Centeno and D. Felbacq, "Optical bistability in finite-size nonlinear bidimensional photonic crystals doped by a microcavity," Phys. Rev. B, vol. 62, pp. R7683-R7686, Sep 2000. [Online]. Available: http://link.aps.org/doi/10.1103/PhysRevB.62.R7683 
[20] M. F. Yanik, S. Fan, and M. Soljai, "High-contrast all-optical bistable switching in photonic crystal microcavities," Appl. Phys. Lett., vol. 83, no. 14, pp. 2739-2741, 2003. [Online]. Available: http://scitation.aip.org/content/aip/journal/apl/83/14/10.1063/1.1615835

[21] S. F. Mingaleev, A. E. Miroshnichenko, Y. S. Kivshar, and K. Busch, "All-optical switching, bistability, and slow-light transmission in photonic crystal waveguide-resonator structures," Phys. Rev. E, vol. 74, p. 046603, Oct 2006. [Online]. Available: http://link.aps.org/doi/10.1103/PhysRevE.74.046603

[22] S. F. Mingaleev, A. E. Miroshnichenko, and Y. S. Kivshar, "Lowthreshold bistability of slow light in photonic-crystal waveguides," Opt. Express, vol. 15, no. 19, pp. 12380-12385, Sep 2007. [Online]. Available: http://www.opticsexpress.org/abstract.cfm?URI=oe15-19-12380

[23] G. Stegeman, G. Assanto, R. Zanoni, C. Seaton, E. Garmire, A. Maradudin, R. Reinisch, and G. Vitrant, "Bistability and switching in a nonlinear prism coupling," Appl. Phys. Lett., vol. 52, no. 11, pp. 869-871, MAR 141988.

[24] G. Assanto, B. Svensson, D. Kuchibhatla, U. J. Gibson, C. T. Seaton, and G. I. Stegeman, "Prism coupling into zns waveguides: a classic example of a nonlinear coupler," Opt. Lett., vol. 11, p. 644, 1986.

[25] J. Ehrlich, G. Assanto, and G. Stegeman, "Butterfly bistability in grating coupled thin-film wave-guides," Opt. Commun., vol. 75, no. 5-6, pp. 441-446, MAR 151990.

[26] G. Assanto, J. Ehrlich, and G. Stegeman, "Feedback-enhanced bistability in grating coupling into insb wave-guides," Opt. Lett., vol. 15, no. 8, pp. 411-413, APR 151990

[27] K. Nozaki, A. Shinya, S. Matsuo, Y. S. T. Segawa, T. Sato, Y. Kawaguchi, R. Takahashi, and M. Notomi, "Ultralow-power alloptical ram based on nanocavities," Nat. Photon., vol. 6, pp. 248-252, 2012.

[28] V. R. Almeida and M. Lipson, "Optical bistability on a silicon chip," Opt. Lett., vol. 29, no. 20, pp. 2387-2389, Oct 2004. [Online]. Available: http://ol.osa.org/abstract.cfm?URI=ol-29-20-2387

[29] L. J. X. Dai and Y. Xiang, "Low threshold optical bistability at terahertz frequencies with graphene surface plasmons," Sci. Rep., vol. 5, no. $12271,2015$.

[30] I. V. Shadrivov, K. Y. Bliokh, Y. P. Bliokh, V. Freilikher, and Y. S. Kivshar, "Bistability of anderson localized states in nonlinear random media," Phys. Rev. Lett., vol. 104, p. 123902, 2010.

[31] J. E. Bjorkholm, A. E. Kaplan, P. W. Smith, and W. J. Tomlinson, "Optical bistability based on self-focusing," Opt. Lett., vol. 6, no. 7, pp. 345-347, Jul 1981. [Online]. Available: http://ol.osa.org/abstract.cfm?URI=ol-6-7-345

[32] J. E. Bjorkholm and A. A. Ashkin, "cw self-focusing and self-trapping of light in sodium vapor," Phys. Rev. Lett., vol. 32, no. 4, pp. 129-132, 1974.

[33] G. I. Stegeman and M. Segev, "Optical Spatial Solitons and Their Interactions: Universality and Diversity," Science, vol. 286, no. 5444, pp. 1518-1523, 1999.

[34] Y. S. Kivshar and G. P. Agrawal, Optical Solitons. San Diego, CA: Academic, 2003.

[35] A. E. Kaplan, "Bistable solitons," Phys. Rev. Lett., vol. 55, pp. 12911294, 1985.

[36] N. Kravets, A. Piccardi, A. Alberucci, O. Buchnev, M. Kaczmarek, and G. Assanto, "Bistability with optical beams propagating in a reorientational medium,” Phys. Rev. Lett., vol. 113, p. 023901, Jul 2014. [Online]. Available: http://link.aps.org/doi/10.1103/PhysRevLett.113.023901

[37] A. Piccardi, N. Kravets, A. Alberucci, O. Buchnev, and G. Assanto, "Voltage-driven beam bistability in a reorientational uniaxial dielectric," APL Photonics, vol. 1, no. 1, 2016.

[38] H. L. Ong, "Optically induced freedericksz transition and bistability in a nematic liquid crystal," Phys. Rev. A, vol. 28, pp. 2393-2407, 1983.

[39] P. G. DeGennes and J. Prost, The Physics of Liquid Crystals. New York: Oxford Science, 1993.

[40] S.-T. Wu and D.-Y. Kang, Fundamental of Liquid Crystal Devices. New York: Wiley, 2006.

[41] I. C. Khoo, Liquid Crystals: Physical Properties and Nonlinear Optical Phenomena. New York: Wiley, 1995.

[42] N. Tabiryan and B. Zeldovich, "The orientational optical nonlinearity of liquid-crystals," Mol. Cryst. Liq. Cryst., vol. 62, pp. 237-250, 1980.

[43] I. C. Khoo, "Nonlinear optics of liquid crystalline materials," Phys. Rep., vol. 471, pp. 221-267, 2009.

[44] G. Assanto, A. Fratalocchi, and M. Peccianti, "Spatial solitons in nematic liquid crystals: from bulk to discrete," Opt. Express, vol. 15, no. 8 , pp. 5248-5259, 2007.
[45] G. Assanto and M. Karpierz, "Nematicons: self-localized beams in nematic liquid crystals," Liq. Cryst., vol. 36, p. 1161, 2009.

[46] M. Peccianti and G. Assanto, "Nematicons," Phys. Rep., vol. 516, pp. $147-208,2012$.

[47] O. Bang, W. Krolikowski, J. Wyller, and J. J. Rasmussen, "Collapse arrest and soliton stabilization in nonlocal nonlinear media," Phys. Rev. E, vol. 66, p. 046619, 2002.

[48] C. Conti, M. Peccianti, and G. Assanto, "Route to nonlocality and observation of accessible solitons," Phys. Rev. Lett., vol. 91, p. 073901 , 2003.

[49] N. S. A.S. Zolot'ko, V.F. Kitaeva and A. Sukhorukovi, "Self-focusing of laser radiation in the course of the freedericksz transition in the nematic phase of a liquid crystal," JETP, vol. 54, no. 3, p. 496, 1981.

[50] I. C. Khoo, "Optical bistability in nematic films utilizing self-focusing of light," Appl. Phys. Lett., vol. 41, no. 10, pp. 909-911, 1982.

[51] A. J. Karn, S. M. Arakelian, Y. R. Shen, and H. L. Ong, "Observation of magnetic-field induced first-order optical Fréedericksz transition in a nematic film," Phys. Rev. Lett., vol. 57, pp. 448-451, 1986.

[52] S.-H. Chen and J. J. Wu, "Observation of first-order Fréedericksz transition in a nematic film induced by electric and optical fields," Appl. Phys. Lett., vol. 52, no. 23, pp. 1998-2000, 1988.

[53] M. G. Clerc, T. Nagaya, A. Petrossian, S. Residori, and C. S. Riera, "First-order Fréedericksz transition and front propagation in a liquid crystal light valve with feedback," Eur. Phys. J. D, vol. 28, pp. 435445, 2004.

[54] E. A. Babayan, I. A. Budagovsky, S. A. Shvetsov, M. P. Smayev, A. S. Zolot'ko, N. I. Boiko, and M. I. Barnik, "Light- and electric-fieldinduced first-order orientation transitions in a dendrimer-doped nematic liquid crystal," Phys. Rev. E, vol. 82, p. 061705, 2010.

[55] I. A. Budagovsky, D. S. Pavlov, S. A. Shvetsov, M. P. Smayev, A. S. Zolot'ko, N. I. Boiko, and M. I. Barnik, "First-order light-induced orientation transition in nematic liquid crystal in the presence of lowfrequency electric field," Appl. Phys. Lett., vol. 101, no. 2, p. 021112 , 2012

[56] F. Simoni, D. E. Lucchetta, L. Lucchetti, H. L. Ong, S. V. Serak, and N. Tabiryan, "First-order optical Fréedericksz transition in a dye-doped nematic liquid crystal," Opt. Lett., vol. 38, no. 6, pp. 878-880, 2013.

[57] A. Alberucci, A. Piccardi, M. Peccianti, M. Kaczmarek, and G. Assanto, "Propagation of spatial optical solitons in a dielectric with adjustable nonlinearity," Phys. Rev. A, vol. 82, no. 2, p. 023806, 2010.

[58] V. Nersesyan, T. Brans, F. Beunis, R. Drampyan, J. Beeckman, and K. Neyts, "Light-controlled reorientation of nematic liquid crystal driven by an electric field," Liq. Cryst., vol. 43, no. 10, pp. 1422-1430, 2016.

[59] A. Alberucci and G. Assanto, "Propagation of optical spatial solitons in finite-size media: interplay between nonlocality and boundary conditions," J. Opt. Soc. Am. B, vol. 24, no. 9, pp. 2314-2320, 2007.

[60] A. Alberucci, A. Piccardi, N. Kravets, and G. Assanto, "Beam hysteresis via reorientational self-focusing," Opt. Lett., vol. 39, no. 20, pp. 5830-5833, Oct 2014. [Online]. Available: http://ol.osa.org/abstract.cfm?URI=ol-39-20-5830

[61] A. Alberucci, A. Piccardi, N. Kravets, O. Buchnev, and G. Assanto, "Soliton enhancement of spontaneous symmetry breaking," Optica, vol. 2, no. 9, pp. 783-789, Sep 2015.

[62] D. W. McLaughlin, D. J. Muraki, M. J. Shelley, and X. Wang, "A paraxial model for optical self-focussing in a nematic liquid crystal," Physica D, vol. 88, no. 1, pp. $55-81,1995$.

[63] M. Peccianti, A. D. Rossi, G. Assanto, A. D. Luca, C. Umeton, and I. C. Khoo, "Electrically assisted self-confinement and waveguiding in planar nematic liquid crystal cells," Appl. Phys. Lett., vol. 77, no. 1, pp. 7-9, 2000.

[64] J. Beeckman, K. Neyts, X. Hutsebaut, C. Cambournac, and M. Haelterman, "Simulations and experiments on self-focusing conditions in nematic liquid-crystal planar cells," Opt. Express, vol. 12, no. 6, pp. 1011-1018, Mar 2004. [Online]. Available: http://www.opticsexpress.org/abstract.cfm?URI=oe-12-6-1011

[65] A. W. Snyder and D. J. Mitchell, "Accessible solitons," Science, vol. 276, p. 1538, 1997.

[66] C. Conti, M. Peccianti, and G. Assanto, "Observation of optical spatial solitons in a highly nonlocal medium," Phys. Rev. Lett., vol. 92, p. 113902, 2004.

[67] S. Ouyang, Q. Guo, and W. Hu, "Perturbative analysis of generally nonlocal spatial optical solitons," Phys. Rev. E, vol. 74, no. 3, p. 036622 , 2006.

[68] A. Alberucci, C. P. Jisha, and G. Assanto, "Accessible solitons in diffusive media," Opt. Lett., vol. 39, pp. 4317-4320, 2014.

[69] A. Alberucci, J. C. P., and G. Assanto, "Nonlinear negative refraction in reorientational soft matter," Phys. Rev. A, vol. 92, p. 033835, 2015. 
[70] E. Braun, L. P. Faucheux, and A. Libchaber, "Strong self-focusing in nematic liquid crystals," Phys. Rev. A, vol. 48, no. 1, pp. 611-622, Jul 1993.

[71] M. Peccianti, A. Fratalocchi, and G. Assanto, "Transverse dynamics of nematicons," Opt. Express, vol. 12, p. 6524, 2004.

[72] O. Buchnev, A. Piccardi, M. Kaczmarek, and G. Assanto, "Nematicon waveguides: self-confined beams and their electric control," Appl. Phys. B, vol. 108, no. 1, pp. 177-182, 2012. [Online]. Available: http://dx.doi.org/10.1007/s00340-012-4978-9

[73] A. Alberucci, N. Kravets, A. Piccardi, O. Buchnev, M. Kaczmarek, and G. Assanto, "Nematicons in planar cells subject to the optical fréedericksz threshold," Opt. Express, vol. 22, no. 25, pp. 30663-30668, Dec 2014.

[74] E. P. Raynes, R. J. A. Tough, and K. A. Davies, "Voltage dependence of the capacitance of a twisted nematic liquid crystal layer," Mol. Cryst. Liq. Cryst., vol. 56, pp. 63-68, 1979.

[75] J. Li, C.-H. Wen, S. Gauza, R. Lu, and S.-T. Wu, "Refractive indices of liquid crystals for display applications," J. Display Technol., vol. 1, pp. 51-61, 2005.

[76] M. Liu, Y. Sun, D. A. Powell, I. V. Shadrivov, M. Lapine, R. C. McPhedran, and Y. S. Kivshar, "Nonlinear response via intrinsic rotation in metamaterials," Phys. Rev. B, vol. 87, p. 235126, Jun 2013. [Online]. Available: http://link.aps.org/doi/10.1103/PhysRevB.87.235126

[77] A. A. Zharov, A. A. Zharov, and N. A. Zharova, "Spontaneous reorientations of meta-atoms and electromagnetic spatial solitons in a liquid metacrystal," Phys. Rev. E, vol. 90, p. 023207, Aug 2014. [Online]. Available: http://link.aps.org/doi/10.1103/PhysRevE.90.023207

[78] M. Peccianti, C. Conti, and G. Assanto, "The interplay between non locality and nonlinearity in nematic liquid crystals," Opt. Lett., vol. 30, p. 415, 2005.

[79] A. Alberucci and G. Assanto, "Nematicons beyond the perturbative regime," Opt. Lett., vol. 35, no. 15, pp. 2520-2522, 2010. 